\title{
Regional differences and trends in breast cancer surgical procedures and their relation to socioeconomic disparities and screening patterns
}

\author{
Christian Herrmann $^{1,2,3}$ (D) Silvia Ess ${ }^{1} \cdot$ Esther Walser $^{1} \cdot$ Harald Frick $^{4} \cdot$ Beat Thürlimann $^{5,6}$ - Nicole Probst-Hensch ${ }^{2,3}$. \\ Christian Rothermundt ${ }^{5}$. Penelope Vounatsou ${ }^{2,3}$
}

Received: 3 August 2018 / Accepted: 3 December 2018 / Published online: 6 January 2019

(C) The Author(s) 2019

\begin{abstract}
Background Important regional disparities in patterns of care in breast cancer have recently been described. In Switzerland, nationwide data on hospitalisations have been collected since 1998. They have not been used up to now to explore space-time patterns and trends of breast cancer healthcare-related procedures for control and health planning purposes. We aimed to assess geographical and temporal variation of mastectomy rates.

Methods Bayesian negative-binomial spatio-temporal models have been applied. Covariates included patient characteristics as provided in the hospital data, data on mammography screening programme duration, and surgeon and gynaecologist density.

Results We analyzed more than 70,000 patients. Mastectomy rates declined from 43\% to 30\% in Switzerland between 2000 and 2012 for patients aged 50-69 and from $61 \%$ to $43 \%$ for those 70+, and remained stable for those under 50. Important geographical differences in rates were present. Rates were significantly influenced by age [relative rate ratio (RR) 50-69: 0.92, RR 70+: 1.25], differences in co-morbidity (RR one co-morbidity: 1.17, RR more than one: 1.35), higher surgeon or gynaecologist density (RR surgeons: 1.01, RR gynaecologists: 1.06). Regions in the French-speaking part (RR: 0.72) and/or with mammography screening programmes showed significantly lower rates (RR: 0.87). No difference was found for patients in different socioeconomic groups or with different insurance types.

Conclusion This research unveiled important differences in mastectomy rates in Switzerland. The results play an essential role in the identification of regions where special attention is required, and indications for extensive surgery in breast cancer should be revisited.
\end{abstract}

Keywords Public health $\cdot$ Mastectomy $\cdot$ Epidemiology $\cdot$ Breast cancer $\cdot$ Switzerland

Statement

The study is based on the thesis "Geographical disparities, trends and projections in female gender-related cancers in Switzerland" by Christian Herrmann, and can be assessed at https://edoc.unibas.ch/ 64104/1/PhD-thesis-v7-EDOC.pdf.

Electronic supplementary material The online version of this article (https://doi.org/10.1007/s10389-018-01007-7) contains supplementary material, which is available to authorized users.

Christian Herrmann

christian.herrmann@kssg.ch

1 Cancer Registry St. Gallen-Appenzell, Cancer League Eastern Switzerland, St. Gallen, Switzerland

2 Department of Public Health, University of Basel, Basel, Switzerland

3 Swiss Tropical and Public Health Institute, Basel, Switzerland
4 Institute for Pathology at the Cantonal Hospital, St. Gallen, Switzerland

5 Department of Internal Medicine, Division Oncology-Haematology, St. Gallen, Switzerland

6 Breast Centre St. Gallen, Cantonal Hospital, St. Gallen, Switzerland 


\section{Introduction}

Female breast cancer is the most frequently diagnosed cancer in the female Swiss population. In the period of 2010-2014, almost 6000 new cases of breast cancer were diagnosed per year, corresponding roughly to $30 \%$ of the female cancer incidence. Within Switzerland, age-standardised incidence rates for breast cancer vary between 83.1/100,000 PY in Appenzell Innerrhoden and 136.8/100,000 in Jura (NICER 2017). Differences in uptake of screening activities (mammography screening in women) contribute as much to differences in incidence as do other known risk factors such as age and genetic predisposition, overweight and obesity, reproductive factors, and presumably hormone replacement therapy (Kumle 2008; McPherson et al. 2000).

Breast surgery is the cornerstone in the treatment of localized breast cancer, representing $95 \%$ of all newly diagnosed $\mathrm{BC}$ patients. Adjuvant therapies (radiotherapy, endocrine therapy, and chemotherapy) are aimed to reduce the risk of relapse and overall survival. While adjuvant therapies are generally performed in the ambulatory setting, breast surgery is performed in Switzerland in an in-patient setting. Data on this hospital procedure is the basis for this study. Mastectomy has been the traditional type of breast surgery until results from well-designed randomized trials in the 1980s showed, that less mutilating surgical procedures had similar rates of overall survival and disease-free survival to those for mastectomy. Preserving the most part of the breast (breast-conserving surgery) through procedures such as lumpectomy or quadrantectomy was aimed at optimal disease control while preserving the quality of life (Veronesi et al. 1981). The studyupdate with a 20 -year follow-up confirmed the preliminary findings, establishing the concept of breast conservation as a standard of care (Veronesi et al. 2002). In fact, mastectomy patients compared to breast-conserving surgery patients usually reported less positive body image and sexual functioning (Montazeri 2008). There are, however, several reasons to perform a mastectomy in contrast to a breast-conserving surgery (BCS). Reasons might be of a personal, medical, or preventive nature, such as an increased risk of getting a second cancer due to BRCA mutations. Medical reasons include larger tumours, multiple areas of the breast affected by cancer, and inflammatory breast cancer. BCS should in most cases be combined with radiotherapy to result in the same survival as mastectomies (McLaughlin 2013). Mastectomies might therefore also be chosen when radiation therapy is medically contraindicated, after previous BCS with radiation therapy, or on a personal level, if the patient prefers to avoid radiotherapy, e.g., living far from facilities offering radiation therapy (Mac Bride et al. 2013). In comparison with BCS, mastectomy is a more serious procedure that sometimes results in complications such as infection, poor healing, and lymphedema, and requires longer hospital stays (Andersen and Kehlet 2011).
Discomfort and pain are less after BCS, but (timeconsuming) radiation and surveillance by mammography are necessary and might result in higher anxiety about recurrence.

In Switzerland, geo-referenced data on hospitalisations including socioeconomic characteristics of the patient, diagnosis, and procedures have been collected yearly by the Federal Statistical Office (FSO) since 1998 and cover the whole country. To our knowledge, the wealth of information provided in this database has not been fully explored to assess space-time patterns and trends of breast cancer healthcare-related procedures for control and health planning purposes.

There has been much debate on the role of medical care in the discussion of social inequalities in health. Access inequalities to early detection, appropriate care, and state of the art management, as well as differences in tumour biology, are possible explanations for survival differences between socio-economic classes. Regional disparities have been described for Switzerland affecting income, access to services including access to healthcare services, education, and other socioeconomic factors.

Availability of public or private resources to be allocated to health is high. Switzerland is one of the richest countries in the world and has among the highest expenditure per person on health and direct purchasing power adjusted costs for cancer. In Switzerland the standard of care is high, uptake of new drugs is above average within Europe (Jonsson and Wilking 2005), and life expectancy is one of the highest in the world. However, since healthcare policies are mainly developed at the cantonal level, there is a considerable amount of geographical variation in health expenditures, control programmes, and treatment procedures. Opportunistic screening is common, especially in the urban areas of cantons with cancer registries, but little is known about regions not covered by cancer registries (Wanner et al. 2001).

Important regional disparities in the state-of-the-art management of breast cancer among regions covered by cancer registration have recently been published (Ess et al. 2010). Disparities included surgical as well as non-surgical management issues. Predictors of guideline compliance on the patient level were treatment by a surgeon with high caseload, residence, and age of the patient, but not socio-economic factors. They described pronounced differences in mastectomy rates from $24 \%$ in Geneva to $38 \%$ in St. Gallen in 2003-2005. The differences persisted after adjustment for age and tumour size. Using the hospital discharge dataset, we can assess if these differences persisted over time and how their results relate to the situation in the whole country.

The aim of this research is to assess spatio-temporal patterns of mastectomy rates in Switzerland and explore their relationship to socioeconomic disparities and screening patterns. The assessment of the geographical variations in the country will help to identify regions in which special attention is required to reduce healthcare inequalities and their impact on the health of the community. 


\section{Methods}

Data from the Swiss Hospital Discharge database until 2012 was retrieved from the Federal Statistical Office (FSO). The database was initiated in 1998 and includes each inpatient hospitalisation discharge with information on the age, the gender of the patient, year of hospitalisation, diagnosis and comorbidities, treatment procedures, class of stay, and administrative characteristics. Its data is available for research in anonymised form in two variants, where we used the geographic variant with information on the region of patients' residence and canton of hospitalisation, over the type of hospital. In both cases, the exact hospital cannot be identified. We excluded data before 2000 due to insufficient data quality,. I.e., unique person identifiers were missing in a significant proportion. The FSO described data completeness and quality issues in the first years after the start of the data collection. Although participation is mandatory, only $85 \%$ of hospitals representing 73\% of cases sent data for 1999 (Bundesamt für Statistik 2003). Since 2000, data have continuously improved, reaching $99 \%$ of hospitals representing $91 \%$ of cases in 2002, and reached $100 \%$ in 2011 (BFS 2017). In our study, we included females with breast cancer (BC, ICD-10 codes C50.0-C50.9) as the main reason for hospitalisation, who had at least one BC-related surgery as defined below in any hospital stay, in order to exclude patients solely treated with palliative intentions or BC-unrelated conditions. Patients who presented with distant metastases at first visit (ICD-10 codes C78-C79) were also excluded. Patients were only counted once per year even with multiple admissions, but counted again when they underwent surgical treatment for breast cancer in another year.

The outcome of interest was the (crude) ratio of patients with mastectomies per all included patients and its trend per hospital region (canton). Data on treatments were coded according to the Swiss operation classification (CHOP) of 2012. CHOP codes begin with the letter ' $\mathrm{Z}$ ' followed by two to six numbers in groups of two, separated by a period. Operations involving the breast have codes beginning with "Z85". To identify women with mastectomies we used the codes "Z85.23" and "Z85.33" to "Z85.48". As (therapeutic) surgeries we used additionally the codes "Z85.2", "Z85.20"- "Z85.22", and "Z85.25". Other codes in the "Z85"-group include diagnostic procedures, reconstructions, transplantations, etc.

We assessed the comorbidity score for each patient based on the hospital record. We used the Charlson score with ICD-10 modification, and performed the calculation using the Stata program "charlson". For the score, we excluded the breast cancer and any breast cancer-related axillary lymph node metastases, in order to reflect the impact of additional health disorders and not to mask any further cancer disease.
From the hospital databases, we determined breast cancer patient characteristics for each region and year. After prior analysis, patients were categorised into three age groups: $<50$ years old, 50-69 years old, and more than 70 years old. This coincides with the age of invitation (50-69-year-olds) in Swiss mammography screening programmes. We categorized patients also by number of admissions (one versus multiple $\mathrm{BC}$-related admissions), by number of $\mathrm{BC}$ surgeries (one versus multiple $\mathrm{BC}$ surgeries), the comorbidity score (score of $0=$ only BC vs score of 1 vs scores of 2 or more) and insurance class (basic vs half-private or private insurance). This insurance class is a proxy for affluence but may in part also reflect differences in treatment. While in principle the same medical services are provided in all classes, patients within the private class are normally treated by more senior staff.

We evaluated also a different covariate in relation to socioeconomic status. Data on socio-economic position (SEP) by municipality was provided by the Swiss National Cohort (SNC 2015) based on census data of 2000, including measures of affluence, education, etc. We linked the municipality of residence of the patient to the SEP score of the municipality, and grouped all BC patients by SEP quartile. The score ranged from 55 to 71 , with the median being 64 and the interquartile range being $60-67$.

We furthermore included covariates in relation to the analysis region, the canton. Data on language region were retrieved from the FSO (Swiss Federal Statistical Office 2017). Regions were classified according to their predominant language into German, French, or Italian/Romansh. Patterns of mammography screening were obtained as years of population-based mammography screening programmes by year and canton (swiss cancer screening 2015). The regions were classified according to the existence of a populationbased mammography screening programme for a given year. The number of surgeons and gynaecologists per 1000 population by year and canton was retrieved from the Swiss Medical Association (FMH 2017). The FMH provides numbers only by main medical discipline. Statistics on hospital type and volume were retrieved from the Federal Office of Public Health (FOPH) (BAG 2014). Since some influencing factors described by other countries, such as urbanisation or hospital type profiles, were not available in this study, it can not be ruled out that any of the above mentioned variables could act as a proxy for some unobservable factor. Although this might be the case for any analytical study, we additionally evaluated the overall hospital type and caseload distribution by canton and its relation to differences in mastectomy rates.

In the analysis, the cantons of Uri, Obwalden, and Nidwalden, and of Appenzell Innerrhoden and Appenzell Ausserrhoden were combined in two regions in order to increase power.

We assessed space-time patterns of mastectomy rates by fitting Bayesian areal models with temporal trends. Bayesian 
methods have been applied extensively in recent years for modelling spatial data because they allow flexible modelling and inference, and provide computational advantages via the implementation of Markov chain Monte Carlo (MCMC) methods. The models produce smooth maps of the observed rates, highlighting patterns of the disease. They are also useful in establishing associations between disease rates and potential area-related risk factors, taking into account geographical correlation. By adding a time dimension, Bayesian spatiotemporal models indicate changes of geographical patterns over time, and determine how the disease evolves over time in different regions and different groups of the population. In our model, the choice of AR(1) for the time trend was based on a prior analysis.

To our knowledge, this is the first study which will utilise the Swiss Hospital database to explore space-time patterns of care in oncology research.

Patient characteristics, socio-economic status, screening patterns, linguistic region, and surgeon and gynaecologist density were explored by including these factors as covariates in the model. The geographical unit of analysis was the canton of the hospital.

For each patient characteristics combination (insurance type, SEP quantile, comorbidity, admissions, surgeries) observed counts of mastectomies $Y_{i j t}$ in canton $i(i=1, \ldots, N)$, age group $j$ and year $t$ to follow a negative binomial distribution $Y_{i j t} \sim \operatorname{Neg} \operatorname{Bin}\left(\mu_{i j t}\right)$. Random effects, as well as possible trends, were modelled on the log of the mean mastectomy rate. Time dimension was included as an autoregressive term [AR(1)] conditioned on the mastectomy rates in the year 2000 .

$$
\begin{aligned}
\log \left(\mu_{i j t}\right)= & \log \left(P_{i j t}\right)+\alpha+\sum X_{i t s}^{T} \beta_{s}+\gamma_{j} \\
& +\left(\delta+\varepsilon_{j}\right) \log \left(\mu_{i j(t-1)}\right)+\Phi_{i}
\end{aligned}
$$

where $P_{i j t}$ is the number of patients, $X_{i t s}$ the vector of covariates $s$ related to canton $i$ and year $t, \beta_{s}$ the coefficients of associated covariates, $\gamma_{j}$ effects of age group $j, \delta$ time trend and $\varepsilon_{j}$ interaction of time and age group $j$. Spatial correlation by random effects $\Phi_{i}$ on canton level $i$, modelled via a conditional autoregressive (CAR) process. Spatial dependency among the cantons was introduced by the conditional prior distribution of $\Phi_{i}$ with

$\Phi_{i} \sim N\left(\begin{array}{c}\gamma \sum_{q=1}^{N} c_{i q} \Phi_{q} \\ \frac{q \neq i}{w_{i}}, \frac{\sigma^{2}}{w_{i}}\end{array}\right)$

where $c_{i q}$ indicates the degree of spatial influence of canton $i$ to the remaining cantons, taking the value 1 if they are adjacent and 0 otherwise, and $\gamma$ quantifying the overall spatial dependence and $w_{i}$ being the number of neighbours of canton $i$. The final model was selected by stepwise selection based on lowest DIC (deviance information criterion) score, including comparison of Poisson vs negative binomial distribution and universal vs single spatial random effects.

From the model estimates we produced maps with the relative differences of the estimated (crude) rates for all ages and by age group combined by 4 -year periods compared to the national mean for 2001-2012.

\section{Results}

A total of 71,655 patients were included in our study. Table 1 summarizes the characteristics of included patients: $22 \%$ were aged $<50$ at hospitalisation, about half $50-69$ and $29 \% 70$ or

\begin{tabular}{|c|c|c|}
\hline & Patients & $\%$ \\
\hline \multicolumn{3}{|l|}{ Age } \\
\hline $0-39$ & 3601 & $5 \%$ \\
\hline $40-49$ & 12,325 & $17 \%$ \\
\hline $50-59$ & 16,447 & $23 \%$ \\
\hline $60-69$ & 18,603 & $26 \%$ \\
\hline $70-79$ & 13,332 & $19 \%$ \\
\hline $80+$ & 7347 & $10 \%$ \\
\hline \multicolumn{3}{|c|}{ Language region (place of treatment) } \\
\hline German & 47,200 & $66 \%$ \\
\hline French & 19,291 & $27 \%$ \\
\hline Italian & 5164 & $7 \%$ \\
\hline \multicolumn{3}{|c|}{ Mammography screening duration in 2012 (place of residence) } \\
\hline 0 (no programme) & 54,111 & $76 \%$ \\
\hline $1-4$ years & 5,369 & $7 \%$ \\
\hline $5-9$ years & 6,887 & $10 \%$ \\
\hline $10+$ years & 5,288 & $7 \%$ \\
\hline \multicolumn{3}{|l|}{ Insurance class } \\
\hline 1 basic & 43,505 & $61 \%$ \\
\hline 2 half-private & 18,159 & $25 \%$ \\
\hline 3 private & 9,991 & $14 \%$ \\
\hline \multicolumn{3}{|c|}{ Comorbidity score (excl. breast cancer) } \\
\hline 0 (only BC) & 64,857 & $91 \%$ \\
\hline 1 & 4923 & $7 \%$ \\
\hline $2+$ & 1875 & $3 \%$ \\
\hline \multicolumn{3}{|l|}{ No. of admissions } \\
\hline 1 & 45,546 & $64 \%$ \\
\hline $2-4$ & 24,334 & $34 \%$ \\
\hline $5+$ & 1775 & $2 \%$ \\
\hline \multicolumn{3}{|l|}{ No. of surgeries } \\
\hline 1 & 55,400 & $77 \%$ \\
\hline 2 & 13,717 & $19 \%$ \\
\hline $3+$ & 2538 & $4 \%$ \\
\hline
\end{tabular}

Table 1 Characteristics of patients included in the study 
over. Two-thirds were treated in the German-speaking region, $27 \%$ in the French-speaking region, and $7 \%$ in the Italianspeaking region. Sixty-one percent of patients had mandatory basic insurance, $25 \%$ half-private, and $14 \%$ private insurance. Nine percent of patients had further co-morbidities at hospitalisation, $64 \%$ were only hospitalised once, and $77 \%$ had only one breast cancer-related surgery. Three-quarters of patients lived in a canton with no established mammography screening programme at their time of hospitalisation (Table 1).

Overall crude mastectomy rates decreased from $48 \%$ of all surgically treated women in 2000 to $36 \%$ in 2012 (Fig. 1). The rate and trend for mastectomy rates are quite different for the three age groups studied. While rates for women below 50 years of age are more or less stable around $40 \%$, with an apparent slight increase, rates in both other groups decreased. The strongest decrease can be observed in the 70 or over age group, from $61 \%$ in 2000 to $43 \%$ in 2012. Mastectomy rates in 50-69-year-olds were much lower and decreased at a slower pace, from $43 \%$ in 2000 to $30 \%$ in 2012.

For the Bayesian regression, the DIC was lowest when all available covariates were included in the model.

The rates vary considerably among the cantons. Figure 2 visualises the relative differences in estimated mastectomy rates for all ages combined by 4-year periods compared to the national mean for 2001-2012. Maps with relative differences by age group and periods are presented in section Annex 2 (Figs. 3, 4, and 5). These show that while the overall mastectomy rates stayed high throughout in central Switzerland (UR/OW/NW), rates decrease considerably from high to average in the age group $<50$ years. Geographical differences in 50-69-year-olds and those 70 or over are with the exception of Zürich nearly identical. In 50-69-year-olds nearly all cantons fall in the same relative rate category as the respective canton in the 70 or over group in all time periods, with the exception of the canton Zurich, where a stronger reduction was observed for the 70 or over group. The spatial patterns and trends in the less than 50-year-olds are different from the other two age-groups (see Annex 2, Figs. 3, 4, and 5).

The regression results are presented as rate ratios (RR) and 95\% Bayesian credible intervals (CI) in Table 2. When taking spatial dependence of the analysis regions as well as other confounding factors into account, the time trends included in the model did not show significance.

Statistically important positive effects, increasing the rate of mastectomies, were age 70 or over (RR 1.25, CI:1.211.29), co-morbidities (score of 1: RR 1.17, CI: 1.11-1.25; score of 2+: RR 1.35, CI: 1.27-1.45), surgeon and gynaecologist density (surgeon density: RR 1.01, CI: 1.00 1.02, gynaecologist density: RR 1.06, CI: 1.05-1.06). Significant decreasing effects were the presence of organised mammography screening programmes (RR 0.87, CI: 0.82 $0.95)$, treatment in the French language region (RR 0.72, CI: 0.62-0.82) and age 50-69 (RR 0.92, CI: 0.87-0.95). Not significant, but important for model fit in terms of DIC, were the number of surgeries per patient and SEP quartile and insurance type of patients.

\section{Discussion}

Our study shows considerable geographical differences in the proportion of women that have a mastectomy as the definitive surgical procedure for breast cancer. This difference persists after taking into account differences in age and case mix (comorbidities). In most regions, the rate of mastectomies declined over time or was already low.
Fig. 1 Observed mastectomy rates by time and age group in Switzerland

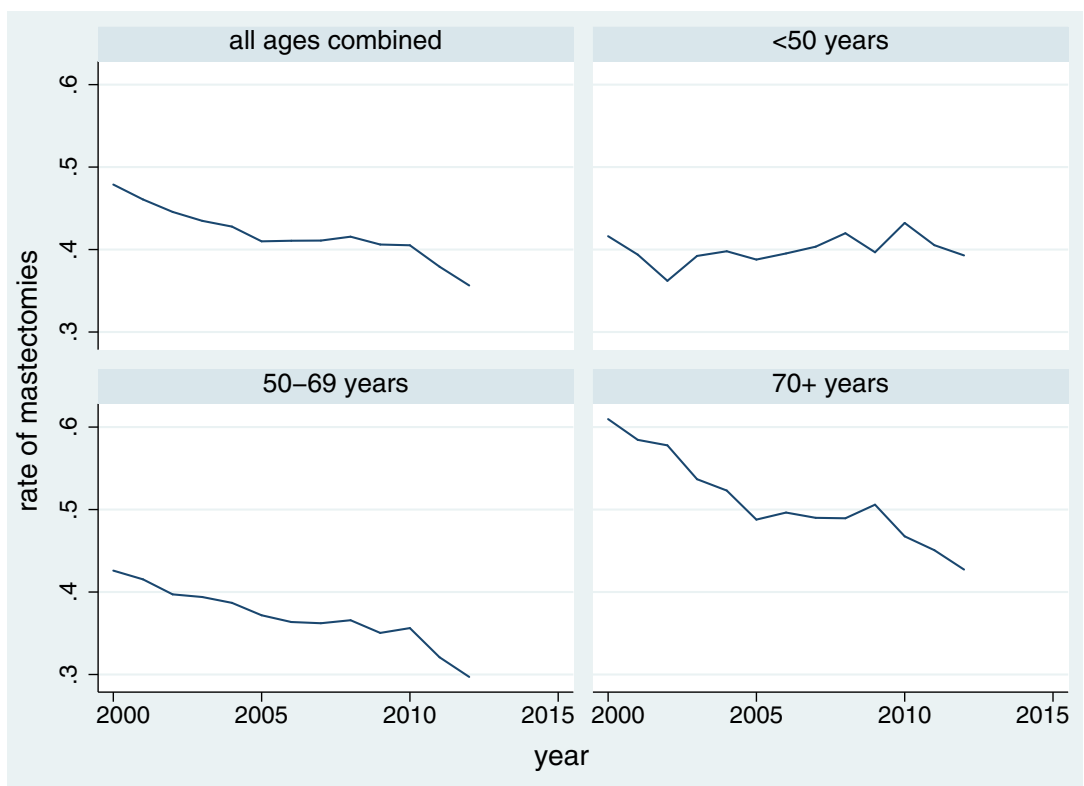


Fig. 2 Estimates of mastectomy rate ratios in Switzerland by time period in relation to the overall mean
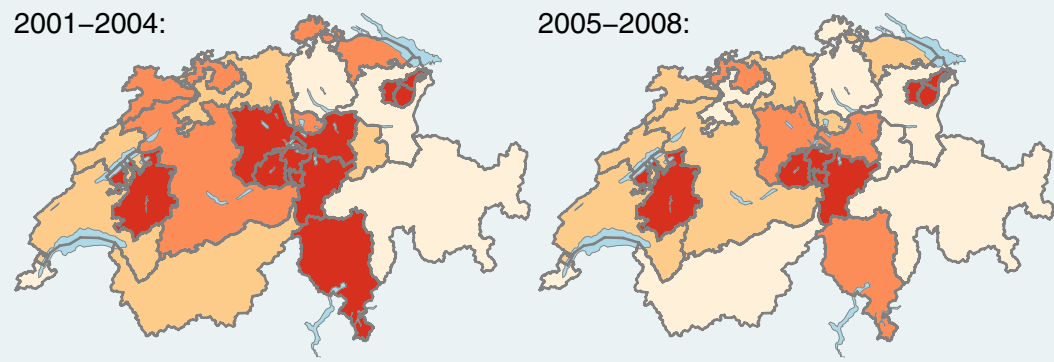

2009-2012:

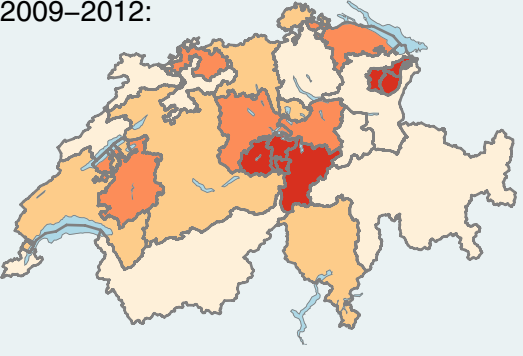

$\mathrm{RR}$ of mastectomies comp. to the overall mean

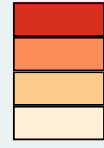

$(1.20,1.45$

$(1.05,1.20]$

$(0.95,1.05]$

$[0.80,0.95]$
We were also able to show the importance of taking spatial dependency and other influencing factors into account, rather than limiting the analysis to age groups. We were able to show that the observed differential trends in the age groups were likely a combined result of trends in all factors. Nevertheless, a significantly higher proportion of $70+$ yearolds received mastectomies compared to $<50$-year-olds, who again had a significantly higher rate of mastectomies than the 50-69-year-olds.

\section{Influence of covariates}

Patients with a higher number of comorbidities had a significantly higher mastectomy rate. This is in line with previous reports. Patients with a higher number of co-morbidities and more severe health conditions tend to receive mastectomies in order to avoid further deteriorating effects of otherwise necessary therapies such as neo-adjuvant chemotherapy and adjuvant radiation (Bernardi et al. 2008).

We adjusted for multiple admissions and multiple breast surgeries per patient. Both factors are strongly overlapping. More admissions/surgeries per patient would suggest that these patients underwent re-excisions and/or mastectomies after a BCS with an insufficient result. More than threequarters of patients in our study received only one surgery, and the variation in this rate was small. However, the inclusion of and adjustment for both characteristics lead to a significantly better model fit. Due to the data structure, we deemed this approach superior to excluding patients with mastectomies after BCS.

We could show that patients were treated similarly in terms of mastectomies regardless of socio-economic group or insurance type. These variables remained in the final model since they increased model fit. In New Zealand also, no differences in mastectomy rates among different socio-economic groups were found when adjusted for several factors. They had no data on insurance type, but described higher mastectomy rates in public versus private hospitals (Seneviratne et al. 2017).

Mastectomy rates in the French language region were significantly lower. This is the language region where mammography screening programmes started the earliest. However, we included the existence of population-based mammography screening programmes in our model, and showed an additional significantly reduced rate of mastectomies of about $13 \%$. In 2001, three cantons had established screening programmes. Up to 2012, ten cantons had had screening programmes for more than 10 years, and three for at least 5 years. Screening programmes lead to a downshift in stage distribution in the respective cantons (Bulliard et al. 2011). And Ess et al. showed a significantly lower rate of mastectomies in Switzerland for breast cancer patients with lower stages (Ess et al. 2010).

While the results in terms of co-morbidities and screening were expected, the other significant covariates indicate possible starting points for reducing geographical disparities. It should be kept in mind that possibly further confounding variables exist, which were or could not be included in the model.

More mastectomies were performed in cantons with more surgeons or gynaecologists per 1000 population. This could have several reasons. On the one hand, those variables may have acted as a proxy for hidden variables not available to this study such as urbanisation or hospital type profiles. Or they may have captured some of the time trends in mastectomy rates, since the rate of surgeons and gynaecologists was also declining at the same time. One 
Table 2 Mastectomy rate ratios, multivariate Bayesian regression results including $95 \%$ credible interval (CI)

\begin{tabular}{|c|c|c|c|}
\hline & Median & $95 \% \mathrm{CI}$ & \\
\hline Time (1-year change) & 1.008 & 1.003 & 1.013 \\
\hline \multicolumn{4}{|l|}{ Time:age group interaction } \\
\hline time: $<50$ years & 1 & (reference) & \\
\hline time: $50-69$ years & 0.995 & 0.987 & 1.001 \\
\hline time: $70+$ years & 0.999 & 0.993 & 1.005 \\
\hline \multicolumn{4}{|l|}{ Age group } \\
\hline$<50$ years & 1 & (reference) & \\
\hline $50-69$ years & $0.915^{*}$ & 0.870 & 0.953 \\
\hline $70+$ years & $1.254 *$ & 1.205 & 1.290 \\
\hline \multicolumn{4}{|l|}{ Co-morbidity score } \\
\hline 0 (only BC) & 1 & (reference) & \\
\hline 1 & $1.171^{*}$ & 1.105 & 1.250 \\
\hline $2+$ & $1.349 *$ & 1.268 & 1.454 \\
\hline \multicolumn{4}{|l|}{ Insurance class } \\
\hline Basic & 1 & (reference) & \\
\hline Half-private/private & 0.982 & 0.951 & 1.015 \\
\hline Multiple admissions & $1.431^{*}$ & 1.033 & 1.790 \\
\hline Multiple BC surgeries & 1.231 & 0.959 & 1.674 \\
\hline \multicolumn{4}{|l|}{ Average SEP at patients' residence } \\
\hline Q1 (lowest quartile) & 1 & (reference) & \\
\hline Q2 & 0.965 & 0.926 & 1.011 \\
\hline Q3 & 0.970 & 0.930 & 1.019 \\
\hline Q4 (highest quartile) & 0.987 & 0.950 & 1.041 \\
\hline \multicolumn{4}{|l|}{ Language region of treatment } \\
\hline German & 1 & (reference) & \\
\hline French & $0.719 *$ & 0.615 & 0.815 \\
\hline Italian/Romansh & 0.878 & 0.792 & 1.013 \\
\hline \multicolumn{4}{|l|}{ Hospital region profiles } \\
\hline Surgeon density per 1000 population & $1.010^{*}$ & 1.002 & 1.015 \\
\hline Gynaecologist density per 1000 population & $1.058^{*}$ & 1.045 & 1.063 \\
\hline Mammography screening programme exists & $0.868^{*}$ & 0.820 & 0.949 \\
\hline Spatial variation & $0.3098^{*}$ & 0.3882 & 0.5353 \\
\hline
\end{tabular}

* denotes significant difference from 1 for covariates. Spatial variation (standard deviation of spatial random effects): a value of 0 means that there is no spatial correlation reason could be that women choose mastectomies more easily if immediate breast reconstruction is available (McLaughlin 2013), i.e., with a higher concentration of plastic surgeons. Also, lower case-volumes have been previously linked to higher mastectomy rates (Hawley et al. 2006). However, it is not possible to conclude a lower case-load from an elevated surgeon or gynaecologist density. In addition, expansion of medical services may lead to performing unnecessary procedures. But whether any of this played a role in the results in relation to the surgeon or gynaecologist density could not be answered by this study, since this type of data was not available.

After adjustment especially for co-morbidities, it is surprising that those over 70 have more mastectomies than 50-69- year-olds. The significantly higher rates in the elderly patients are not observed in a similar magnitude in other countries. Cultural and personal factors may have played a greater role in this age group, but the magnitude is a reason for concern and would need more detailed research.

No overall decline in mastectomy rates was observed in the age group of $<50$-year-olds. This might be due to lower rates a priori and/or a higher proportion of hereditary forms of breast cancer where radical mastectomies of both breasts are combined curative and preventive measures (Rebbeck et al. 2004). However, mastectomy is not universally used for treating hereditary forms of breast cancer and the rate might change over time with reviewed evidence (Paradiso and Formenti 2011). 


\section{Comparison of results with previous publications}

Regional differences in mastectomy rates in 2003-2005 have been previously reported for Switzerland (Ess et al. 2010). Ess et al. analysed data from 11 cantons covering about half of the Swiss population, with the main focus on patterns of breast cancer care. They described significantly different rates of mastectomies among some study regions. When compared with our results, the participating regions in the above study all had lower mastectomy rates in the spectrum of all cantons, with the exception of Appenzell Innerrhoden and Ausserrhoden.

Trends and influencing factors of mastectomy rates have been studied for various countries. The rates and observed trends vary greatly among the countries. Most studies found a significant decrease in mastectomy rates, with the exception of rates in Alberta, Canada, and France (Fisher et al. 2015; Rococo et al. 2016). Most studies also found significant geographical variation within the country. In the USA (excluding patients above 80 years of age), the trends among the age groups are comparable to our findings, as well as the range of relative differences among the regions ranging from 0.71-times the total average in Connecticut and 1.32-times the average in Louisiana (Habermann et al. 2010). The regional variation in the Netherlands was significant and had a similar magnitude to our findings, with adjusted odds ratios ranging for $<40$-year-olds from 0.7 to 1.49 , for $40-69$-year-olds from 0.65 to 1.45 , and for those 70 or over from 0.65 to 1.85 (van Steenbergen et al. 2010).

\section{Strengths and limitations}

One major limitation of the study is that the hospital data did not include information on the breast cancer stage and no intent of treatment (palliative or curative). Moreover, we could not distinguish the breast cancer by laterality, since this information was only included recently in the dataset and therefore missing for most of the years. In order to reduce a possible bias because of preventive contralateral mastectomy, we only counted one mastectomy per woman.

A second major limitation is that no detailed information about single hospitals was available. For anonymization purposes, the Federal Statistical Office (FSO) pooled all hospitals in a canton in the available dataset, making it impossible to distinguish them from another. Therefore, we had no information about the type of hospital (university, central, rural, private etc.) in which a patient was treated. However, the Swiss cantons are relatively small, and from the general hospital statistic provided by the FSO, we were able to evaluate the overall hospital type and caseload distribution by canton (see Annex, Fig. 6) as a rough proxy of hospital type and caseload for breast cancer patients. Regardless of the grouping of hospital types, no direct correlation with mastectomy rates could be observed at this level of detail. In other countries, both type and hospital volume were described as significant factors for mastectomy rates, e.g., in Finland (Peltoniemi et al. 2011). While this factor might be a key target to approach reducing disparities, our study specifically chose to analyse geographical patterns, since health policy is mainly developed on cantonal level. In particular, our study gives important insight into which areas might benefit most from interventions, i.e., in resource allocation and policy planning.

The surgeon density was only available for the profession as a whole and not by area of expertise or case-load. Therefore, the results and possible implications need further research. Urbanisation could not be used since the hospital data was only available on a cantonal level.

The advantage of the current study is the population-based nature of the database, resulting in a large representative sample of over 70,000 patients included in our study. The results mirror the real-world situation in Switzerland. Bayesian regression models have not been employed until now to explore differences in cancer management.

\section{Conclusion}

This is the first time full benefit was taken from the information of the national hospital discharge database to analyse geographical differences in breast cancer care, in particular mastectomies. Mastectomy rates declined importantly in Switzerland in 2000-2012 for patients aged 50-69 and 70+, and remained stable for those under 50 . Rates were highest for those 70 and above and lowest for those aged 50-69. Regional differences in mastectomy rates are as pronounced in Switzerland as in other countries.

We showed the importance of taking spatial dependence and other influencing factors into account when comparing mastectomy rates among age groups and geographical areas.

Patients from different socio-economic groups or with different insurance types did not receive different treatment with regard to mastectomies. Rates were significantly influenced by differences in co-morbidity. Regions with a higher surgeon and gynaecologist density had higher rates of mastectomies, and regions with mammography screening programmes lower rates. In general, it is important to note that higher or lower than average rates are not the same as "too high" or "too low" since this study is of ecological design. The decision to undergo mastectomy or BCS is ideally done on an individual level by a wellinformed patient, and is based on medical reasons and personal preferences. Both types of surgery have their advantages and disadvantages, but analysing the 
differences in rates and trends may help in gaining a clearer insight into the decision-making process in the surgical treatment of breast cancer. This research unveiled important information which until now was not available for the whole country. Further research is needed to understand the combined role of region-specific and hospital-specific factors, such as hospital type and volume, on breast cancer care differences.

Funding $\mathrm{CH}$ was supported by the Cancer League Eastern Switzerland, and $\mathrm{CH}$ and $\mathrm{PV}$ were supported by a grant of the Swiss National Science Foundation with the number 32003B_135769. The funders had no role in the study design, data collection, and analysis, decision to publish, or preparation of the manuscript.

\section{Compliance with ethical standards}

Conflict of interests The authors declare that they have no conflict of interest. CR reports institutional support outside the submitted work for "Consulting or Advisory Role" from Novartis, Pfizer, Astellas Pharma, Bristol-Myers Squibb, Eisai, and PharmaMar.

Open Access This article is distributed under the terms of the Creative Commons Attribution 4.0 International License (http:// creativecommons.org/licenses/by/4.0/), which permits unrestricted use, distribution, and reproduction in any medium, provided you give appropriate credit to the original author(s) and the source, provide a link to the Creative Commons license, and indicate if changes were made.

Publisher's note Springer Nature remains neutral with regard to jurisdictional claims in published maps and institutional affiliations.

\section{References}

Andersen KG, Kehlet H (2011) Persistent pain after breast cancer treatment: a critical review of risk factors and strategies for prevention. J Pain 12:725-746. https://doi.org/10.1016/j.jpain.2010.12.005

BAG (2014) Kennzahlen der Schweizer Spitäler = Chiffres-clés des hôpitaux suisses = Cifre chiave degli ospedali svizzeri 2012 . Kennzahlen der Schweizer Spitäler = Chiffres-clés des hôpitaux suisses $=$ Cifre chiave degli ospedali svizzeri. BAG, Bern

Bernardi D, Errante D, Gallligioni E, Crivellari D, Bianco A, Salvagno L, Fentiman IS (2008) Treatment of breast cancer in older women. Acta Oncol 47:187-198. https://doi.org/10.1080/ 02841860701630234

BFS (2017) Medizinische Statistik der Krankenhäuser. Bundesamt für Statistik. https://www.bfs.admin.ch/bfs/de/home/statistiken/ gesundheit/erhebungen/ms.html. Accessed 09.11.2017 2017

Bulliard JL, Ducros C, Dayer E, Arzel B, Levi F (2011) Variation in performance in low-volume mammography screening programmes: experience from Switzerland. Cancer Epidemiol 35:293-297. https://doi.org/10.1016/j.canep.2010.07.015

Ess S, Savidan A, Frick H, Rageth C, Vlastos G, Lutolf U, Thurlimann B (2010) Geographic variation in breast cancer care in Switzerland. Cancer Epidemiol 34:116-121. https://doi.org/10.1016/j.canep. 2010.01.008

Fisher S, Gao H, Yasui Y, Dabbs K, Winget M (2015) Treatment variation in patients diagnosed with early stage breast cancer in Alberta from 2002 to 2010: a population-based study. BMC Health Serv Res 15: 35. https://doi.org/10.1186/s12913-015-0680-z
FMH (2017) FMH Ärztestatistik. Swiss Medical Association. https://www.fmh.ch/services/statistik/aerztestatistik.html. Accessed 30.10.2017

Habermann EB, Abbott A, Parsons HM, Virnig BA, Al-Refaie WB, Tuttle TM (2010) Are mastectomy rates really increasing in the United States? J Clin Oncol 28:3437-3441. https://doi.org/10. 1200/jco.2009.27.6774

Hawley ST, Hofer TP, Janz NK, Fagerlin A, Schwartz K, Liu L, Deapen D, Morrow M, Katz SJ (2006) Correlates of between-surgeon variation in breast cancer treatments. Med Care 44:609-616. https://doi. org/10.1097/01.mlr.0000215893.01968.f1

Jonsson B, Wilking N (2005) A pan-European comparison regarding patient access to cancer drugs. Karolinska Institutet in collaboration with Stockholm School of Economics, Stockholm

Kumle M (2008) Declining breast cancer incidence and decreased HRT use. Lancet 372:608-610. https://doi.org/10.1016/S0140-6736(08) 61255-6

Mac Bride MB, Neal L, Dilaveri CA, Sandhu NP, Hieken TJ, Ghosh K, Wahner-Roedler DL (2013) Factors associated with surgical decision making in women with early-stage breast cancer: a literature review. J Women's Health (Larchmt) 22:236-242. https://doi.org/ 10.1089/jwh.2012.3969

McLaughlin SA (2013) Surgical management of the breast: breast conservation therapy and mastectomy. Surg Clin North Am 93:411428. https://doi.org/10.1016/j.suc.2012.12.006

McPherson K, Steel CM, Dixon JM (2000) ABC of breast diseases. Breast cancer-epidemiology, risk factors, and genetics. BMJ 321: 624-628

Montazeri A (2008) Health-related quality of life in breast cancer patients: a bibliographic review of the literature from 1974 to 2007. J Exp Clin Cancer Res 27:32. https://doi.org/10.1186/1756-9966-27-32

NICER (2017) National Institute for Cancer Epidemiology and Registration. http://www.nicer.org. Accessed 30.01.2017 2017

Paradiso A, Formenti S (2011) Hereditary breast cancer: clinical features and risk reduction strategies. Ann Oncol 22(Suppl 1):i31-i36. https://doi.org/10.1093/annonc/mdq663

Peltoniemi P, Peltola M, Hakulinen T, Hakkinen U, Pylkkanen L, Holli K (2011) The effect of hospital volume on the outcome of breast cancer surgery. Ann Surg Oncol 18:1684-1690. https://doi.org/10.1245/ s10434-010-1514-1

Rebbeck TR, Friebel T, Lynch HT, Neuhausen SL, van 't Veer L, Garber JE, Evans GR, Narod SA, Isaacs C, Matloff E, Daly MB, Olopade OI, Weber BL (2004) Bilateral prophylactic mastectomy reduces breast cancer risk in BRCA1 and BRCA2 mutation carriers: the PROSE study group. J Clin Oncol 22:1055-1062. https://doi.org/ 10.1200/JCO.2004.04.188

Rococo E, Mazouni C, Or Z, Mobillion V, Koon Sun Pat M, Bonastre J (2016) Variation in rates of breast cancer surgery: a national analysis based on French hospital episode statistics. Eur J Surg Oncol 42:5158. https://doi.org/10.1016/j.ejso.2015.09.020

Seneviratne S, Scott N, Lawrenson R, Campbell I (2017) Ethnic, sociodemographic and socio-economic differences in surgical treatment of breast cancer in New Zealand. ANZ J Surg 87:E32-E39. https:// doi.org/10.1111/ans.13011

SNC (2015) Swiss National Cohort. http://www.swissnationalcohort.ch/. Accessed 28.08.2015

Bundesamt für Statistik (2003) Medizinische Statistik : Resultate 1999 (Standardtabellen). BFS aktuell. OFS/BFS, Neuchâtel

Swiss cancer screening (2015) Schweizerischer Verband der KrebsFrüherkennungsprogramme. http://www.swisscancerscreening.ch/. Accessed 28.08.2015

Swiss Federal Statistical Office (2017) STAT-TAB: Die interaktive Statistikdatenbank. Swiss Federal Statistical Office. http://www. pxweb.bfs.admin.ch/. Accessed 04.10.2017 2017

van Steenbergen LN, van de Poll-Franse LV, Wouters MW, JansenLandheer ML, Coebergh JW, Struikmans H, Tjan-Heijnen VC, 
van de Velde CJ (2010) Variation in management of early breast cancer in the Netherlands, 2003-2006. Eur J Surg Oncol 36(Suppl 1):S36-S43. https://doi.org/10.1016/j.ejso.2010.06.021

Veronesi U, Saccozzi R, Del Vecchio M, Banfi A, Clemente C, De Lena M, Gallus G, Greco M, Luini A, Marubini E, Muscolino G, Rilke F, Salvadori B, Zecchini A, Zucali R (1981) Comparing radical mastectomy with quadrantectomy, axillary dissection, and radiotherapy in patients with small cancers of the breast. N Engl J Med 305:6-11. https://doi.org/10.1056/NEJM198107023050102
Veronesi U, Cascinelli N, Mariani L, Greco M, Saccozzi R, Luini A, Aguilar M, Marubini E (2002) Twenty-year follow-up of a randomized study comparing breast-conserving surgery with radical mastectomy for early breast cancer. N Engl J Med 347:1227-1232. https://doi.org/10.1056/NEJMoa020989

Wanner P, Raymond L, Bouchardy C (2001) Geographical disparities in self-reported use of mammography and breast self-examination according to the Swiss health survey. Ann Oncol 12:573-574 